\title{
Intervention in anaphylaxis: the experience of one paediatric centre based on NORA reports
}

\author{
"'Przyladek Zdrowia”, Krakow, Poland \\ 2Department of Paediatrics, Jagiellonian University Medical College, Krakow, Poland \\ 3"Dziupla” Statistical Analysis, Krakow, Poland
}

Izabela Tarczoń1, Urszula Jedynak-Wąsowicz², Grzegorz Lis², Tomasz Tomasik², Piotr Brzyski³ ${ }^{3}$ Ewa Cichocka-Jarosz ${ }^{2}$

Adv Dermatol Allergol 2021; XXXVIII (2): 235-243

DOI: https://doi.org/10.5114/ada.2019.89715

\begin{abstract}
Introduction: Intramuscular adrenaline administration is the primary intervention in anaphylaxis.

Aim: To analyse the data on intervention in children admitted due to anaphylaxis to the tertiary paediatric centre and compare them to the data from the Network for Online-Registration of Anaphylaxis.

Material and methods: A validated structured on-line questionnaire was used to collect data concerning the firstand second-line intervention in anaphylaxis. The study was conducted in cooperation with the European Anaphylaxis Registry.

Results: The study group comprised 114 children (76 boys, 66.87\%) aged 5 months-17 years with the predominance of moderate-to-severe anaphylaxis (grade III in Ring and Messmer's, and grade IV in Mueller's scale). In 103 (90.4\%) children the first line of medical intervention was provided by medical staff. In the first-line intervention 39 (34.8\%) children were given adrenaline. Five (4.4\%) children were given the second dose of adrenaline and were admitted to the intensive care unit. In the second-line intervention adrenaline was given to $12(15.6 \%)$ children. In one third it was at least the second reaction to the same trigger. Children treated with adrenaline were older (9.3 \pm 4.8 years), in comparison to those not treated $(7.3 \pm 4.6$ years, $p=0.034)$. Directly after the episode of anaphylaxis the children got the prescription for the adrenaline autoinjector in 35.1\%, emergency training in $7.9 \%$, and counselling on the avoidance of the anaphylaxis trigger in $30.7 \%$. Grade III R\&M reaction increased 3-fold the odds of AAI prescription (95\% Cl: 1.08-8.15).
\end{abstract}

Conclusions: There is a strong need to continue education on proper management of anaphylaxis in children.

Key words: anaphylaxis, medical intervention, adrenaline, register, children.

\section{Introduction}

The Network for Online-Registration of Anaphylaxis (NORA, https://www.anaphylaxis.net) was created in 2011 based on the Anaphylaxis Registry for German-language Countries established in 2006 [1]. A continuous collaboration of 10 European countries and Brazil results in collection of anonymous detailed data regarding anaphylactic events in adults and children [2, 3]. The NORA registry is based on the on-line questionnaire. Analysis of the submitted data enables identification of the most common allergens causing anaphylaxis in different age groups, severity of the reactions as a function of the causing agent, influence of comorbidities on development of anaphylaxis [1-3]. Furthermore, evaluation of the above database helps verify compliance of the pro- vided treatment with the current standards and determine whether there is a need for targeted educational provisions.

Even though according to the international standards, the primary intervention in anaphylaxis in all age groups is prompt intramuscular administration of adrenaline into the middle of the vastus lateralis muscle [4-9], it is still not a common procedure [10]. It might be due to difficulties with proper anaphylaxis diagnosis or anxiety associated with the drug use [11]. In addition, neither paediatric nor adult patients receive prescription for an adrenaline autoinjector (AAI) for self-administration [12]. The obvious need for increased education in this regard justifies clinical evaluation of this issue.

Address for correspondence: Ewa Cichocka-Jarosz MD, PhD, Assoc. Prof., Department of Paediatrics, Jagiellonian University Medical College, 265 Wielicka St, 30-663 Krakow, Poland, phone/fax: +48 1265820 11, e-mail: mijarosz@cyf-kr.edu.pl Received: 1.09.2019, accepted: 25.09.2019. 


\section{Aim}

The primary aim of this study is to present data, including type of medical intervention during anaphylaxis and preventive measures afterwards, submitted by the paediatric tertiary referral centre specialized in allergy in the Southern Poland to the NORA Anaphylaxis Registry. The secondary aim is to identify main issues associated with anaphylaxis treatment and prevention, which require improvement and education.

\section{Material and methods}

The study was conducted in cooperation with the European Anaphylaxis Registry. There were included all children admitted during an anaphylaxis episode and these admitted for planned diagnostics following anaphylaxis in the preceding 12 months. The online questionnaires were filled out between September 2015 and March 2019. All the parents, caregivers and youths aged 16-17 provided their written consents prior to their entering the study. The study was approved by the Ethics Committee of the Jagiellonian University (K/ZDS/006266).

The tool of our study was a validated structured online questionnaire.

The factors analysed both in the first- and secondline treatment included the type of an individual that provided intervention, type of medication including adrenaline, and preventive measures following anaphylaxis. The first-line treatment was defined as emergency first aid until stabilization was achieved, or in case it was not achieved, until advanced resuscitation was commenced. The second-line treatment was defined as treatment following stabilization to prevent biphasic or secondary events, or in the event of lack of response to the first-line treatment.

\section{Statistical analysis}

Qualitative data were presented as counts and percentages. Quantitative data were given as means and standard deviations (SD) for normally distributed variables and medians and quartiles otherwise. Normality of quantitative variables was tested using the KolmogorovSmirnov test and inspection of box-plot and Q-Q plot. Between group comparisons of qualitative variables were analysed by $\chi^{2}$ test; when expected frequencies in at least $20 \%$ of cells were lower than 5 , the exact Fisher test was used for $2 \times 2$ tables and Fisher-Freeman-Halton $\chi^{2}$ test in the other cases. Comparisons of quantitative variables between two groups were conducted with Student $t$ test for independent samples for variables of normal distribution and using Mann-Whitney test otherwise. The logistic regression model was used to evaluate the impact of severity of symptoms on adrenaline use; as well as to evaluate the impact of age, gender, elicitor, severity and use of adrenaline in the $1^{\text {st }}$ line interven- tion on adrenaline prescription after the anaphylaxis episode. The results were presented as odds ratios along with respective $95 \%$ confidence intervals and respective $p$-values. A $p$-value of $<0.05$ was considered significant. IBM SPSS Statistics 24 for Windows was used for statistical analysis.

\section{Results}

\section{Characteristics of the study group}

There were data on 126 children entered by our centre to the NORA Registry. The Registry's administrators positively verified questionnaires of 114 (90.5\%) children (study group) aged 5 months to 17 years, without differences in age distribution between genders (Table 1). The predominant anaphylaxis triggers were insect venom and food. The main elicitor of anaphylaxis was food in children less than 3 years of age (68\%), insect venom and food in children aged $4-18$ years (57\% and $27 \%$, respectively). Fifty nine children (51.8\%) had comorbid atopic diseases of the respiratory tract and skin, or food allergy (Table 1). Atopic diseases had been previously diagnosed in $72.5 \%$ of children who developed food elicited anaphylaxis, and $40 \%$ of children with anaphylaxis due to insect venom. Thirty seven and a half percent of children previously diagnosed with food allergy developed anaphylaxis elicited by food. The severity of the allergic reaction was predominantly grade III according to Ring and Messmer's (R\&M) scale, and grade IV according to Mueller's scale (Table 1).

In 36 (31.6\%) children, it was at least the second episode of anaphylaxis elicited by the same trigger, such as insect in $47.2 \%(n=17)$, food in $44.4 \%(n=16)$, allergen immunotherapy, other (grass pollen) and unknown $-2.8 \%$ each $(n=1)$.

\section{First-line intervention}

In 112 out of 114 children (98.2\%) pharmacologic treatment was introduced (Figure 1). Ninety one children $(81.3 \%)$ were hospitalized. In the majority of children, the first line of medical intervention was provided exclusively by medical staff, one fourth received their treatment from a non-medical person first, followed by the medical staff (Figure 1). In a non-medical group, a family member provided treatment in 36 out of 38 cases (94.7\%), a school teacher in 1 case and one child used AAI. The first-line treatment provided by the medical staff in 103/112 (92\%) cases was almost equally done by either an emergency doctor or a non-allergy specialist, followed by a general practitioner and an emergency healthcare professional (paramedic) (Figure 2). The pharmacological intervention performed by the non-medical staff included mainly oral antihistamines, followed by oral GCS and beta-agonists (Figure 1). The pharmacotherapy given by the medical staff was based mostly on the intramuscular GCS, fol- 
lowed by i.v. fluids. About one third of children received adrenaline, usually administered intramuscularly. Medical personnel slightly less frequently than adrenaline administered antihistamines (AH1) orally and intravenously (Figure 1).

Overall, 39 out of 112 children (34.8\%) were given adrenaline as the first-line rescue treatment. Five children (4.4\%) required the second dose of adrenaline during anaphylaxis due to food allergy $(n=2)$, drug allergy $(n=1)$ or an unknown trigger $(n=2)$. These children were admitted to the ICU. Almost all (94.9\%) adrenaline injections as the first-line treatment were administered by the healthcare professionals. Healthcare professionals differed in regards to administration of oral $\mathrm{AH} 1$ ( $p=0.001)$, inhaled $\beta_{2}$-agonists $(p=0.044)$, and oral glucocorticosteroids (GCS) ( $p=0.017)$.

There were 36 (31.6\%) children who developed secondary reactions to the same allergen, out of which one did not receive any treatment and 15 (45.9\%) were administered adrenaline as their first-line treatment. In the latter group, 2 (5.6\%) children allergic to insect venom received adrenaline using AAI (one done by the patient, one done by a teacher), all the other patients were administered adrenaline by medical staff.

Children treated with adrenaline were older $(9.3 \pm 4.8$ years) in comparison to those not treated $(7.3 \pm 4.6$ years, $p=0.034)$. Frequency of adrenaline use was similar irrespective of Mueller's grade in almost all age groups. Only adolescents over 13 years old received adrenaline over 3 times more often in Mueller's grade IV than grade II/III reaction (Figure $3 \mathrm{~A}$ ). According to the R\&M classification, adrenaline was given to 15 (38.5\%) children with R\&M grade II, and 24 (32.9\%) children with R\&M grade III (Figure 3 B). Grade IV according to Mueller scale almost doubled a statistical odds of adrenaline administration

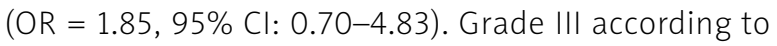
$R \& M$ decreased odds of adrenaline administration by about $1 / 5$ (OR $=0.78,95 \% \mathrm{Cl}$ : 0.35-1.76). Atopy did not increase the risk of adrenaline injection.

\section{Second-line intervention}

In 77/112 treated children (68.8\%), the second-line treatment was needed, which was performed exclusively by the medical staff. Seventy-one (92.2\%) of these children were hospitalized. In over half of the children, it was administered by a non-allergy specialist, followed by an allergy specialist or an emergency doctor. Only in a few cases, the second-line treatment was performed by a general practitioner (Figure 2).

The most common second-line pharmacological intervention included antihistaminics $\mathrm{AH} 1$, both oral and parenteral, followed by GCS, both oral and parenteral, and fluids i.v. (Figure 1).

Adrenaline, as the second-line intervention, was administered to 12 (15.6\%) children (Figure 1).
Table 1. Basic characteristics of the study population

\begin{tabular}{|c|c|c|}
\hline Parameter & $N$ & $\%$ \\
\hline Total & 114 & 100 \\
\hline Male & 76 & 66.7 \\
\hline \multicolumn{3}{|l|}{ Age: } \\
\hline Infants and small children ( $\leq 2$ years) & 17 & 14.9 \\
\hline Preschool children (3-5 years) & 32 & 28.1 \\
\hline School children (6-11 years old) & 40 & 35.1 \\
\hline Adolescents (12-17 years old) & 25 & 21.9 \\
\hline \multicolumn{3}{|l|}{ Elicitors: } \\
\hline Food & 40 & 35.1 \\
\hline Drug & 6 & 5.3 \\
\hline Venom & 54 & 47.4 \\
\hline Allergen immunotherapy & 3 & 2.6 \\
\hline Others & 4 & 3.5 \\
\hline Unknown & 7 & 6.1 \\
\hline \multicolumn{3}{|l|}{ Comorbidities: } \\
\hline Allergic rhinitis & 49 & 43 \\
\hline Asthma & 16 & 14 \\
\hline Atopic dermatitis & 11 & 9.6 \\
\hline Food allergy & 18 & 15.8 \\
\hline \multicolumn{3}{|l|}{ Severity grade: } \\
\hline \multicolumn{3}{|l|}{ According to Ring \& Messmer: } \\
\hline II & 39 & 34.2 \\
\hline III & 75 & 65.8 \\
\hline IV & 0 & 0 \\
\hline \multicolumn{3}{|l|}{ According to Mueller: } \\
\hline II & 1 & 0.9 \\
\hline III & 28 & 24.6 \\
\hline IV & 85 & 74.6 \\
\hline \multicolumn{3}{|l|}{ Year of reported reaction: } \\
\hline 2014 & 28 & 24.6 \\
\hline 2015 & 21 & 18.4 \\
\hline 2016 & 28 & 24.6 \\
\hline 2017 & 23 & 20.2 \\
\hline 2018 & 14 & 12.3 \\
\hline
\end{tabular}

In twenty-three (29.9\%) children, reported anaphylaxis was a repeated response to the same, previously identified allergen. Two of them (8.7\%), allergic to insect venom, received adrenaline. Overall 20 children (22\%) were hospitalized. In Mueller's classification, adrenaline was given to $3(18.8 \%)$ children with grade III and 9 (14.8\%) children with grade IV anaphylaxis. In R\&M's classification, adrenaline was given to $3(10.7 \%)$ children with grade II and 9 (18.4\%) children with grade III symp- 


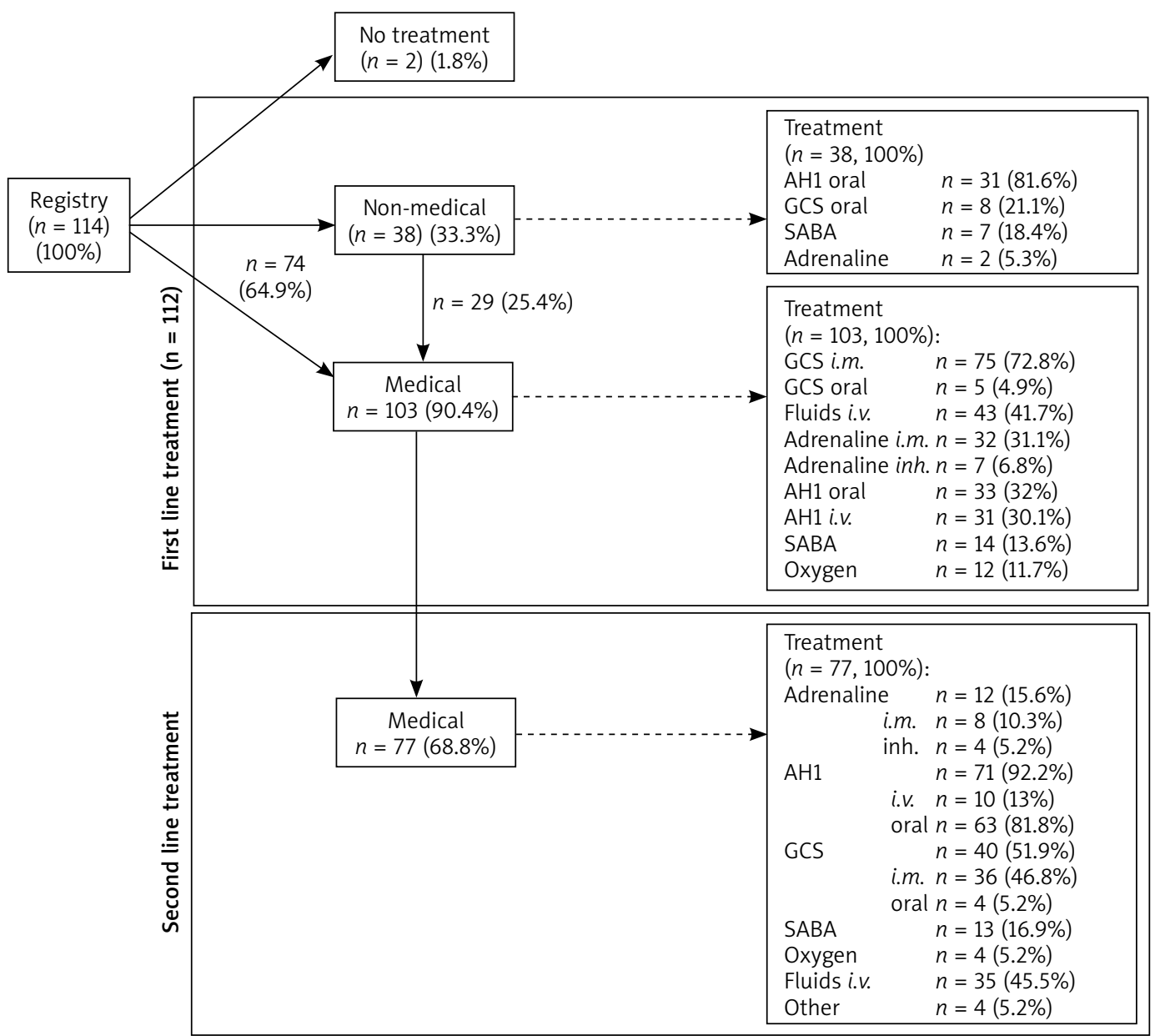

Figure 1. First and second line of pharmacological intervention

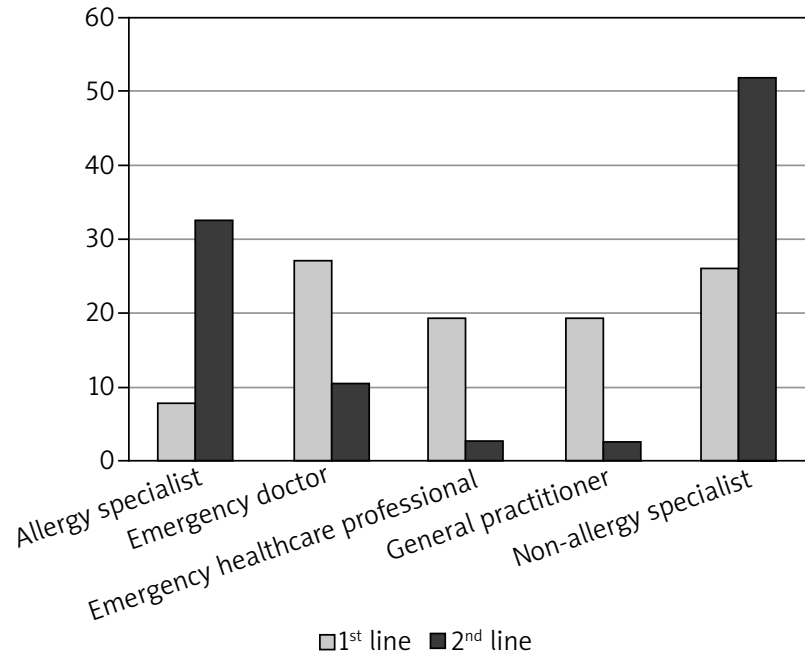

Figure 2. Types of medical specialties providing first- and second-line interventions toms. In the second-line intervention, grade IV according to Mueller scale decreased probability of adrenaline injection by $1 / 4(\mathrm{OR}=0.75,95 \% \mathrm{Cl}$ : 0.18-3.17), on the other hand, grade III according to R\&M scale almost doubled probability of the adrenaline injection $(\mathrm{OR}=1.88,95 \% \mathrm{Cl}$ : 0.46-7.60). Atopy did not increase probability of adrenaline injection.

\section{Preventive measures}

Prior to the reaction reported in the NORA registry, 7 (6.14\%) children were supplied with AAI due to their previous episode of anaphylaxis with confirmed allergy to either insect sting $(n=3)$ or food $(n=4)$. Immediately after the episode of anaphylaxis, one third of children received prescriptions for $A A I$, more frequently than for antihistamines and oral GCS (Figure 4 A). During subsequent follow-up visits, more than one tenth of children got their prescriptions for AAI from their GPS. Following specialist allergy work-up, almost half of children were prescribed AAls, together with oral antihistamines and oral GCS (Figure 4 A). 
A

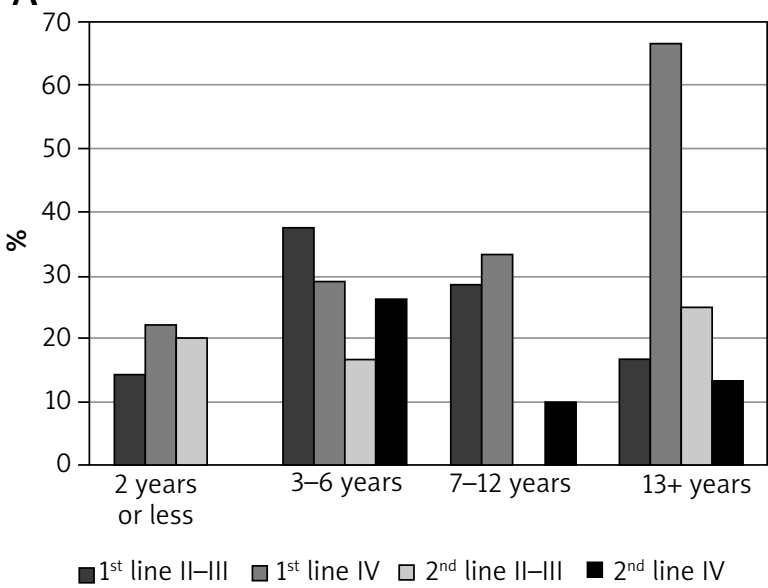

B

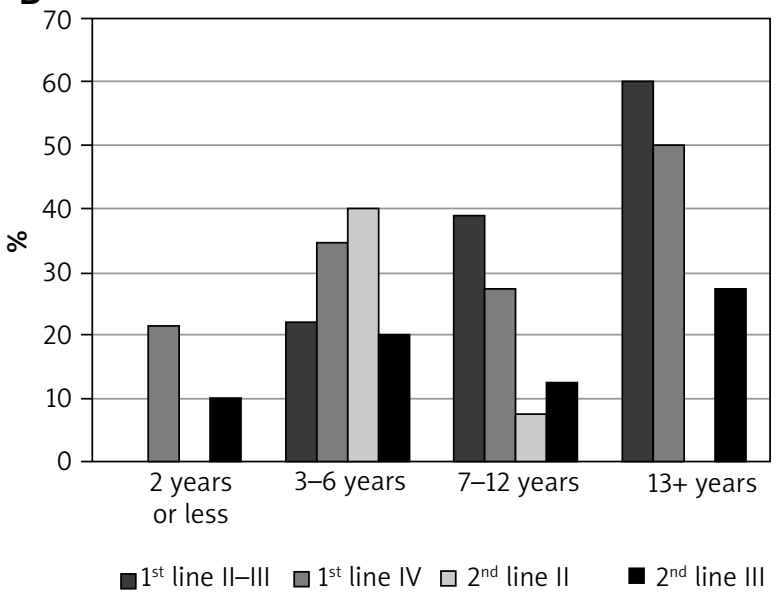

Figure 3. Severity of the reaction according to Mueller's grade (A) and R\&M's grade (B) with respect to age vs. adrenaline in the first- and second-line interventions

Patients and their caregivers were rarely provided with training in emergency management even though they received AAI prescriptions at emergency care or at primary care follow-up visit (Figure 4 B). During the allergy specialist visit, the frequency of training in emergency management was high, though lower than the number of prescriptions for emergency drugs. Specific immunotherapy (in 100\% to insect venom) was provided exclusively by the allergy specialists. Counselling on the avoidance of the anaphylaxis trigger was provided directly after the incident in about one third of patients, during the allergy specialist visit in over half of cases. Only few GPs performed such counselling during follow-up visits (Figure 4 B).

\section{Multivariate analysis \\ Before the visit at the allergy centre}

The children allergic to insect venom had much higher chance to receive prescriptions for AAl in comparison to the ones allergic to food or to allergens other than food or insect venom. Boys had over two times higher chances, however insignificant, to receive AAI prescriptions, as well as older children aged 7 to 12 years compared to the younger ones, up to 3 years old (Table 2 ). Just the fact of adrenaline administration during an anaphylaxis episode showed borderline influence on a non-allergy specialist's decision to prescribe adrenaline. The severity of anaphylaxis according to Mueller's scale did not affect a nonspecialist doctor to prescribe a child an AAI (Table 2).

Table 2. Multivariate analysis of factors influencing AAI prescription after an episode of anaphylaxis

\begin{tabular}{|c|c|c|c|c|}
\hline \multirow{3}{*}{$\begin{array}{l}\text { Parameter } \\
\text { Number of observations } \\
\text { Parameter }\end{array}$} & \multirow{2}{*}{\multicolumn{2}{|c|}{$\begin{array}{l}\text { Before the visit at the allergy centre } \\
\qquad N=52 / 114\end{array}$}} & \multirow{2}{*}{\multicolumn{2}{|c|}{$\begin{array}{l}\text { During the visits at the allergy centre } \\
\qquad N=46 / 114\end{array}$}} \\
\hline & & & & \\
\hline & $\begin{array}{l}\text { Odds ratio } \\
(95 \% \mathrm{Cl})\end{array}$ & $P$-value & $\begin{array}{l}\text { Odds ratio } \\
(95 \% \mathrm{Cl})\end{array}$ & $P$-value \\
\hline Male sex & $2.40(0.91-6.35)$ & 0.079 & $0.36(0.15-0.90)$ & 0.029 \\
\hline Age (vs. $0-2$ years): & 1 & & 1 & \\
\hline $3-6$ & $1.11(0.25-4.84)$ & 0.891 & $0.38(0.10-1.48)$ & 0.166 \\
\hline $7-12$ & $2.01(0.46-8.85)$ & 0.356 & $0.20(0.05-0.80)$ & 0.023 \\
\hline $13+$ & $1.35(0.27-6.79)$ & 0.714 & $0.33(0.07-1.49)$ & 0.148 \\
\hline Elicitor (vs. venom): & 1 & & 1 & \\
\hline Food & $0.20(0.07-0.57)$ & 0.003 & $1.74(0.62-4.88)$ & 0.289 \\
\hline $\begin{array}{l}\text { Others (including latex, exercise and unknown } \\
\text { causes of anaphylaxis) }\end{array}$ & $0.15(0.04-0.51)$ & 0.002 & $3.12(0.97-10.03)$ & 0.056 \\
\hline Mueller's severity grade IV (vs. III + II) & $0.69(0.24-1.97)$ & 0.484 & $1.28(0.52-4.20)$ & 0.467 \\
\hline Adrenaline use in the first-line treatment & $2.54(0.99-6.49)$ & 0.51 & $0.57(0.23-1.43)$ & 0.230 \\
\hline
\end{tabular}


A

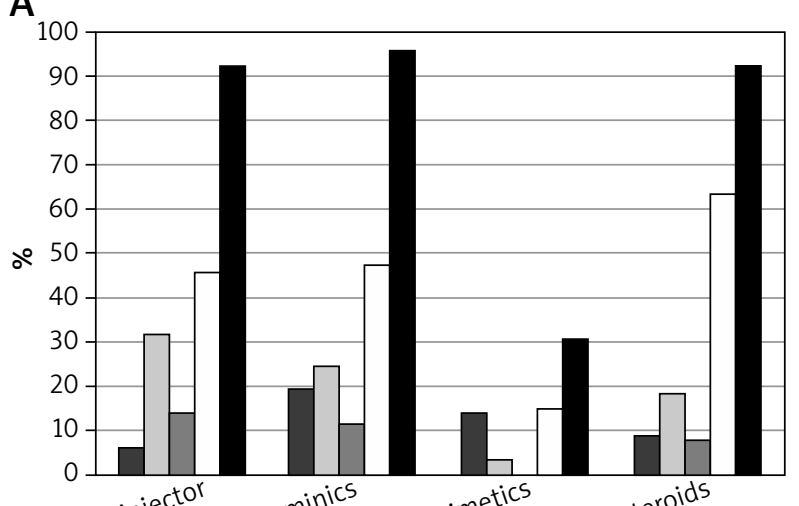

B

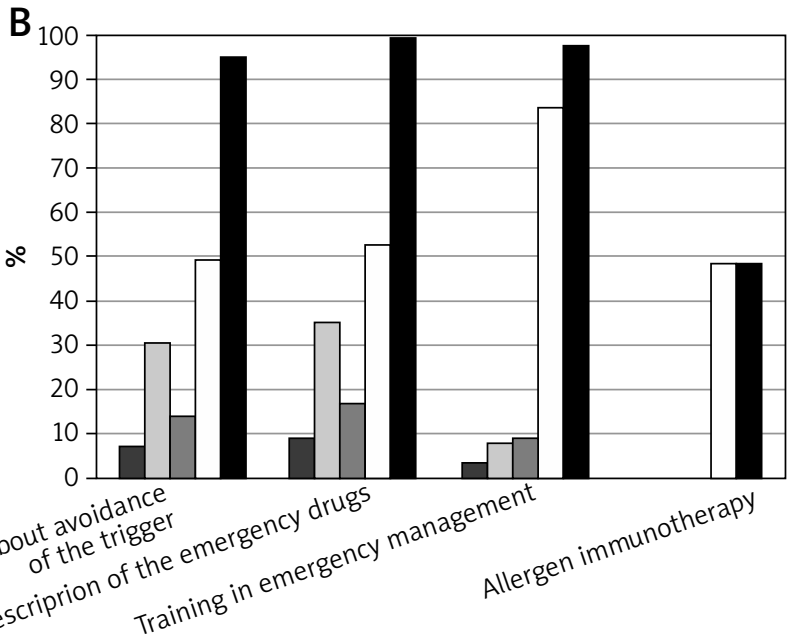

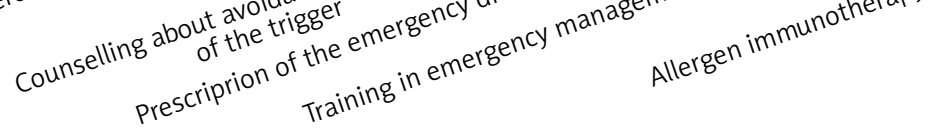

$$
\begin{aligned}
& \text { In primary care as: } \\
& \square \text { Prior to reaction } \\
& \square \text { At the emergency } \\
& \text { situation }
\end{aligned}
$$
Glucocorticosteroids

Figure 4. Pharmacological (A) and general (B) preventive measures taken after an anaphylaxis episode

However when our statistical model included severity of anaphylaxis according to R\&M scale (not Mueller's classification), then there was a predominant effect with borderline significance of gender and severity of anaphylaxis. It appeared that grade III R\&M reaction increased 3 fold (95\% Cl: 1.08-8.15) the odds of AAI prescription regardless of the type of the sensitizing allergen.

\section{During the visit at the allergy centre}

Boys were two and a half times less likely to receive prescriptions for adrenaline. Also, children aged 4 to 18 had smaller odds to obtain prescriptions for adrenaline compared to these younger than 3 years, though this difference was significant only in case of the children aged 7 to 12 years. It appeared that allergy to food doubled and other allergens tripled odds of receiving a prescription for AAl from the allergy specialist; however those differences were not significant (Table 2). Adrenaline administration during emergency treatment did not affect the specialist prescribing AAl. Also, the severity of anaphylaxis did not significantly affect odds of receiving AAI prescription. When the model included severity of anaphylaxis according to R\&M scale, the same factors appeared to determine whether the prescription for AAI was given as compared to the model with Mueller's scale.

\section{Discussion}

\section{Single centre data in comparison to the Registry data}

Currently (as of 4 June 2019), there are accumulated data from 12968 patients in all age groups, including
3680 children and youths in the Anaphylaxis Registry. The number of data from our centre constituted half of the data from Poland $(n=236)$ and $13.3 \%$ of the children collected in the registry of the international centres ( $n=856)$ (https://www.anaphylaxie.net). The severity of their symptoms was determined according to two scales; R\&M's scale typically used in the complete Registry, and Mueller's scale, which was more common in Poland especially in case of venom anaphylaxis. The above scales differed significantly as to types of symptoms assigned to specific grades; therefore, comparisons of studies using those scales were burdensome. There is an increasing need for unification of different scales to analyse anaphylaxis [13].

We included only children with a generalized allergic reaction graded moderate and serious. In the material of the entire registry, over $85 \%$ of the reactions in children and adults were equivalent to severity of grade II and III in R\&M classification, which respectively corresponded to $45 \%$ and $47 \%$ in children $[1,3]$. In the presented material, there were no children presenting grade IV anaphylaxis (circulatory failure, death) according to $R \& M$ scale, while the entire registry reported $0.9 \%$ of children developing grade IV anaphylaxis, including 5 deaths in different age groups and induced by different triggers, such as cow milk, peanuts, hazelnut, cashew, and insect venom [3]. The French registry, Allergy Vigilance Network, also pointed out a death risk in the case of food allergy in children [14].

Analysis of the clinical symptoms in our patients compared to the registry data will be a part of another study. Our observations indicated that allergy to insect venom was the predominant factor inciting anaphylaxis 
since we worked at the tertiary reference centre for diagnostics and immunotherapy for children with allergy to insect venom. However, the majority of the paediatric data including the prospective ones, indicated food as the most common factor triggering anaphylaxis in children [1, 3, 14-18].

Nevertheless, the allergy profile in different age groups of our patients corresponded well with the rest of the registry; food allergy was predominant in the preschool group, insect venom was more prevalent in school children. In the Registry, foods dominated as elicitors of anaphylaxis in the first decade of life, and then they were gradually replaced by insect venom and drugs [3]. Atopy, manifested in the form of atopic dermatitis, atopic allergic rhinitis, asthma, was not a risk factor for more severe anaphylaxis in children according to our data. Registry data for children did not evaluate any significant effect of atopy $[1,3]$.

\section{Intervention during an episode of anaphylaxis}

Our data indicated that the medical intervention was initiated by the medical personnel in more than $90 \%$ of children; among them $64.9 \%$ of children received help exclusively from the medical personnel, mostly emergency doctors. In comparison, the Registry recorded $72 \%$ of children (1363) receiving help from the medical personnel, out which $38 \%$ were assisted by emergency physicians [3]. In our material, the number of interventions provided by the caregivers, mostly parents, corresponded well with the Registry data on children and youth $33.3 \%$ and 30\%, respectively). The number of children applying self-medication was comparable with the data from the German-speaking countries (2.8\% vs. $3 \%)$, but it was lower than in the general Registry that reported $10 \%$ of lay-treated preschoolers and 19\% of adolescents [3, 15]. Adrenaline was administered in total to $42.9 \%$ of children; it was the first-line intervention in about $1 / 3$ of children that developed grade III and IV anaphylaxis according to R\&M's scale. It was higher compared to $28 \%$ in the general Registry, which nevertheless recorded a significant increase in adrenaline use from $12 \%$ to $25 \%$ over the entire time period of data collection [3]. The most common medical assistance included AH1 (89.3\%), GCS i.m. (83\%) and intravenous fluids (61.3\%) in both first and second lines of interventions. In the Registry, treatment with GCS was just as common (82\%), while $\mathrm{AH} 1$ was less common (76\%) [3]. In our study, adrenaline was administered mostly in the form of the intramuscular injection (36.6\%), and additionally as inhalation (9.4\%). We registered neither intravenous nor subcutaneous applications, which were occasionally observed in the German-speaking countries (12 and 2 cases, respectively) [15]. There was a wide divergence in pharmacological interventions during hospitalization with the predominance of systemic GCS (71\%) and AH1 blockers (60\%) according to the US Pediatric Health Information System, which recorded
10,351 children with anaphylaxis [19]. We recorded an increased number of children, $45.9 \%$, receiving adrenaline when symptomatic to the same allergen; however, it was still less than in the Cross-Canada Anaphylaxis Registry in which $66.2 \%$ of children reacting to the known allergic trigger received adrenaline [20]. In our study, 5 (4.4\%) children allergic to food, drug or unknown factor required a second adrenaline dose and hospitalization at the ICU. In the Registry, 26 (1.3\%) children were either admitted to the intensive care unit or developed grade IV/fatal reactions [3]. Those very rare cases should be a reminder that anaphylaxis in children might be serious, even life threatening. However, refractory anaphylaxis resistant to two or more doses of adrenaline were not practically reported in children [21].

Adrenaline administration in only $1 / 3$ of children with severe systemic symptoms underscored its severely limited use in anaphylaxis. It might result from a number of causes, such as insufficient education on anaphylaxis diagnosis, which was pointed out by French authors evaluating the level of familiarity with diagnosis and intervention among 2100 physicians specialized in medical emergency [22]. Another problem might be the fear of side effects, though there were multi-centre studies that demonstrated a high safety profile of adrenaline in all the age groups [23]. There is a limited number of available ready-made adrenaline injections in the medical centres, which typically keep ampoules of $1 \mathrm{mg} /$ $\mathrm{ml}$ adrenaline; hence its administration requires good understanding of dosing, precise execution and proper type of the needle, a tuberculin one. There is a readymade dose of $0.3 \mathrm{mg}$ for children over $25 \mathrm{~kg}$ in the form of either $50 \%$-refunded prefilled syringe, or fully priced, 10 times more expensive adrenaline auto-injector. There is only one ready-made adrenaline autoinjector for children that weigh 7.5-25 kg, which is Epipen Junior with $0.15 \mathrm{mg}$ of adrenaline. It is an expensive product with limited store accessibility, which makes it practically unavailable during emergencies in medical settings. There are also some legal issues in AAl administration [24]. In Poland, the law prohibits any lay person without medical education to administer adrenaline even in life-threatening situations. In other countries, such as the US or Australia, adrenaline is accessible at schools, and there are educational programs that stress the importance of school nurses and teachers' education in the anaphylaxis management $[25,26]$. In Europe, the laws vary among different countries, and each country develops its own recommendations [27]. In Poland, this particular issue is not legally addressed despite attempts by the Polish Allergology Society.

\section{Preventive measures after the episode of anaphylaxis}

In the presented material, the severity of the symptoms justified the prescription for AAl in all the children; however, it was prescribed only in $1 / 3$ of the group. 
The gap in adrenaline supply was filled up by allergy physicians, therefore a total of $90 \%$ children got the prescription. Our data were supported by the Registry data, which demonstrated that $91 \%$ of children were prescribed intervention medications, including $90 \%$ for adrenaline, though there were differences in prescription rates among different severity grades and types of allergens [3]. The incident of adrenaline use during anaphylaxis increased the chance for receiving (future) adrenaline prescription by 2.5 times, borderline statistically significant. The European data matched American observations, in which factors in favour of AAI prescription were adrenaline administration at ED $(O R=3.6$; 95\% Cl: $1.6-7.9 ; p=0.001)$ and insect venom allergy $(\mathrm{OR}=4.0 ; 95 \% \mathrm{Cl}: 1.6-10.5, p=0.004)$ [28].

Once treated for anaphylaxis, the patients typically visited their primary physicians for further management including referral to an allergy specialist. That opened up another possibility to receive AAI prescription in case of not having AAI prescribed earlier, especially that the waiting time for the specialist visit was typically long In the presented material, only several percent of physicians prescribed adrenaline to a paediatric patient. It appeared to be a worldwide issue, also observed by other Polish authors with respect to venom anaphylaxis in adults [27, 29]. The data summarized indicated that physicians performing first intervention presented limited familiarity with anaphylaxis, recommended management during acute symptoms and follow-up visits. According to French data accumulated from 2010 to 2015 at ED, there was only $1 / 3$ of patients referred to the allergist following an anaphylaxis episode [30]. The data evaluated in our questionnaire did not answer this question. In our material, 100\% of children with insect venom severe and moderate grade anaphylaxis initiated immunotherapy, compared to $73 \%$ of children in the general Registry [3]. There was a strikingly low, less than $10 \%$, incidence of patient's education on the anaphylaxis management following patient stabilization and during the follow-up visit with the general practitioners. Even during specialist visits, our data showed that the number of educated children and their caregivers was lower than the number of adrenaline prescriptions. Another important issue was the cost and refund of the medicine. The high price despite experts' recommendations might be prohibitive and result in lack of drug purchase, leading to its unavailability during anaphylaxis. The Swedish authors also raised this particular issue [31]. We strongly agree with conclusions of other authors that a central anaphylaxis registry should be established in Poland as the only approach that would allow for collecting a wide range of reliable clinical information of anaphylaxis [32].

\section{Strengths and limitations of the study}

The strength of our study stems from a relatively large size of the group of children with anaphylaxis in the
Polish population. In addition, our data were compiled in a uniform and systematic fashion since there were only two assistants collecting all the data based on the structured questionnaires. Our data supported the data of the entire Registry, even though they represented only several percentages of the international observations.

A potential limitation of the study might be predominance of the children with insect venom allergy that might result from our status as the reference centre for the management of insect venom anaphylaxis in children. However, we did not find any evidence of such bias in Polish epidemiologic studies.

\section{Conclusions}

Adrenaline was not administered to $2 / 3$ of children with severe anaphylaxis. Directly after an anaphylaxis episode, only $1 / 3$ of patients were prescribed AAl, and less than $10 \%$ were trained in anaphylaxis management following emergency situations or during follow-up visits with their GPs. There is a need to continue education on the diagnosis and management of anaphylaxis. It is critical to make physicians aware of the necessity to educate their patients on the indications and techniques of adrenaline administration, including self-injection.

\section{Acknowledgments}

English proofreader: Anna Knapp, MD, PhD.

\section{Conflict of interest}

The authors declare no conflict of interest.

\section{References}

1. Worm M, Eckermann O, Dölle S, et al. Triggers and treatment of anaphylaxis: an analysis of 4,000 cases from Germany, Austria and Switzerland. Dtsch Arztebl Int 2014; 111: 367-75.

2. Worm M, Moneret-Vautrin A, Scherer K, et al. First European data from the network of severe allergic reactions (NORA). Allergy 2014; 69: 1397-404.

3. Grabenhenrich LB, Dölle S, Moneret-Vautrin A, et al. Anaphylaxis in children and adolescents: the European Anaphylaxis Registry. J Allergy Clin Immunol 2016; 137: 1128-37.e1.

4. Muraro A, Roberts G, Worm M, et al. Anaphylaxis: guidelines from the European Academy of Allergy and Clinical Immunology. Allergy 2014; 69: 1026-45.

5. Muraro A, Lemanske RF Jr, Castells M, et al. Precision medicine in allergic disease-food allergy, drug allergy, and anaphylaxis-PRACTALL document of the European Academy of Allergy and Clinical Immunology and the American Academy of Allergy, Asthma and Immunology. Allergy 2017; 72: 1006-21.

6. Simons FE, Ardusso LR, Bilò MB, et al. International consensus on (ICON) anaphylaxis. World Allergy Organ J 2014; 7: 9.

7. Simons FE, Ebisawa M, Sanchez-Borges M, et al. 2015 update of the evidence base: World Allergy Organization anaphylaxis guidelines. World Allergy Organ J 2015; 8: 32 
8. Ring J, Beyer K, Biedermann T, et al. Guideline for acute therapy and management of anaphylaxis: S2 Guideline of the German Society for Allergology and Clinical Immunology (DGAKI), the Association of German Allergologists (AeDA), the Society of Pediatric Allergy and Environmental Medicine (GPA), the German Academy of Allergology and Environmental Medicine (DAAU), the German Professional Association of Pediatricians (BVKJ), the Austrian Society for Allergology and Immunology (ÖGAI), the Swiss Society for Allergy and Immunology (SGAI), the German Society of Anaesthesiology and Intensive Care Medicine (DGAI), the German Society of Pharmacology (DGP), the German Society for Psychosomatic Medicine (DGPM), the German Working Group of Anaphylaxis Training and Education (AGATE) and the patient organization German Allergy and Asthma Association (DAAB). Allergo J Int 2014; 23: 96-112.

9. Available at: https://www.nice.org.uk/guidance/qs119/chapter/List-of-quality-statements.

10. Grabenhenrich LB, Dölle S, Ruëff F, et al. Epinephrine in severe allergic reactions: The European Anaphylaxis Register. J Allergy Clin Immunol Pract 2018; 6: 1898-06.e1.

11. Song TT, Worm M, Lieberman P. Anaphylaxis treatment: current barriers to adrenaline auto-injector use. Allergy 2014; 69: 983-91.

12. Owusu-Ansah S, Badaki O, Perin J, et al. Under prescription of epinephrine to medicaid patients in the Pediatric Emergency Department. Glob Pediatr Health 2019; 6: $2333794 X 19854960$.

13. Muraro A, Fernandez-Rivas M, Beyer K, et al. The urgent need for a harmonized severity scoring system for acute allergic reactions. Allergy 2018; 73: 1792-800.

14. Pouessel G, Cerbelle V, Lejeune S, et al. Anaphylaxis admissions in pediatric intensive care units: follow-up and risk of recurrence. Pediatr Allergy Immunol 2019; 30: 341-7.

15. Hompes S, Köhli A, Nemat K, et al. Provoking allergens and treatment of anaphylaxis in children and adolescents: data from the anaphylaxis registry of German-speaking countries. Pediatr Allergy Immunol 2011; 22: 568-74.

16. Cohen N, Capua T, Pivko D, et al. Trends in the diagnosis and management of anaphylaxis in a tertiary care pediatric emergency department. Ann Allergy Asthma Immunol 2018; 121: 348-52.

17. Ruiz Oropeza A, Lassen A, Halken S, et al. Anaphylaxis in an emergency care setting: a one year prospective study in children and adults. Scand J Trauma Resusc Emerg Med 2017; 25: 111.

18. Ross MP, Ferguson M, Street D, et al. Analysis of food allergic and anaphylactic events in the National Electronic Injury Surveillance System. J Allergy Clin Immunol 2008; 121: 166-71.

19. Michelson KA, Monuteaux MC, Neuman MI. Variation and trends in anaphylaxis care in United States Children's Hospitals. Acad Emerg Med 2016; 23: 623-7.

20.O'Keefe A, Clarke A, St Pierre Y, et al. The risk of recurrent anaphylaxis. J Pediatr 2017; 180: 217-21.

21. Francuzik W, Dölle S, Worm M. Risk factors and treatment of refractory anaphylaxis - a review of case reports. Expert Rev Clin Immunol 2018; 14: 307-14.

22. Lejeune S, Deschildre A, Beaudouin E, et al. Pre-hospital management of pediatric anaphylaxis by French Emergency Medicine physicians: still to be improved. Clin Exp Allergy 2019; 49: 1047-50.

23. Cardona V, Ferré-Ybarz L, Guilarte M, et al. Safety of adrenaline use in anaphylaxis: a multicentre register. Int Arch Allergy Immunol 2017; 173: 171-7.
24. Cicutto L, Julien B, Li NY, et al. Comparing school environments with and without legislation for the prevention and management of anaphylaxis. Allergy 2012; 67: 131-7.

25. Greenhawt M, Wallace D, Sublett JW, et al. Current trends in food allergy-induced anaphylaxis management at school. Ann Allergy Asthma Immunol 2018; 121: 174-8.

26. Vale S, Netting MJ, Ford LS, et al. Anaphylaxis management in Australian schools: review of guidelines and adrenaline autoinjector use. J Paediatr Child Health 2019; 55: 143-151.

27. Tham EH, Leung ASY, Pacharn P, et al. Anaphylaxis - lessons learnt when East meets West. Pediatr Allergy Immunol 2019 Jun 20. doi: 10.1111/pai.13098.

28. Campbell RL, Luke A, Weaver AL, et al. Prescriptions for self-injectable epinephrine and follow-up referral in emergency department patients presenting with anaphylaxis. Ann Allergy Asthma Immunol 2008; 101: 631-6.

29. Chciałowski A, Abramowicz M, Kruszewski J. Analysis of Hymenoptera venom allergy in own material. Clinical evaluation of reactions following stings, in patients qualified for venom immunotherapy. Adv Dermatol Allergol 2019; 36: 302-7.

30.Dubus JC, Lê MS, Vitte J, et al. Use of epinephrine in emergency department depends on anaphylaxis severity in children. Eur J Pediatr 2019; 178: 69-75.

31. Protudjer JLP, Middelveld R, Ballardini N, et al. Epinephrine dispensings, allergy hospitalizations and the elimination of co-payments in Sweden. Allergy 2019; 74: 1197-200.

32. Jahnz-Rozyk K, Raciborski F, Śliwczyński AM, et al. Anaphylaxis in Poland: the epidemiology and direct costs. Adv Dermatol Allergol 2017; 34: 573-9. 